\title{
Use of thermographic imaging to study babies sleeping at home
}

\author{
E S Anderson, M P Wailoo, S A Petersen
}

\begin{abstract}
Two 3 month old infants sleeping under different thermal conditions were found to maintain similar deep body temperatures. Thermographic imaging suggested that though the uncovered head is the main source of heat transfer, other parts of the body such as the hands may be used when necessary.
\end{abstract}

The deep body temperatures of normal 3 month old babies fall during sleep at night in a pattern that is maintained irrespective of room temperature or thermal insulation of clothing and wrapping. ${ }^{1}$ By this age, therefore, the thermoregulatory abilities of the babies is highly efficient, but many are kept under such warm conditions that heat loss may be compromised. ${ }^{2}$

In this study we examined how thermoregulation is achieved by comparing measurements of body temperature and thermographic images of two babies who maintained almost identical rectal temperature patterns in different thermal environments. As thermographic images give excellent information about surface temperatures we aimed to assess the role of the exposed parts such as head and hands in thermoregulation in the home environment, which is quite different from that in the laboratory. ${ }^{3}$

\section{Methods}

While recording the body temperature of babies sleeping in their cots at home we have been able to make infrared thermographic recordings on a few occasions.

Babies taking part in a larger study were recruited by health visitors, and parents were informed that the purpose of the study was to investigate the body temperature of babies at home. On the evening that the recording was to take place a health visitor fitted the baby with probes that recorded rectal temperature, abdominal skin temperature, and skin temperature on the forehead. The parents then prepared the infant for bed in the usual way, and the probes were attached to a Grant Squirrell Data Logger set to log readings every minute throughout the night. A further probe recorded the temperature in the bedroom. Note was taken of the clothing and wrapping on the baby, so that a 'tog' value could be calculated, and full details of the birth and family circumstances were recorded.

An Insight Vision Systems 80S thermal imaging camera was mounted on a tripod to record the sleeping baby's head and the top part of its Accepted 1 June 1990

(ArchDisChild 1990;65:1266-7). wrappings. The camera black level was set so that mid range colour coding corresponded to $32^{\circ} \mathrm{C}$, with a span of $2^{\circ} \mathrm{C}$. The images were continuously recorded on a video recorder for the first three hours after bedtime, and individual video frames were captured and printed for illustration.

\section{Results}

Body temperature was recorded and thermal imaging carried out on five babies, four of whom slept in conditions similar for ambient temperature and insulation, and who showed similar patterns of deep body temperature and thermal imaging. Case 1 is an example of these. Case 2 slept in much hotter conditions, and made a different thermogenic response.

\section{CASE REPORTS}

Case 1

This baby was born at full term weighing 3100 $\mathrm{g}$, the first son of an engineer and a medical practitioner, and was breast fed. The family lived in their own semidetached house with full gas central heating. The recordings were made in April when he was 3.5 months of age. He slept in his own room with a thermostatically controlled radiator. He wore a disposable napkin, long vest, and 'Babygro' (tog count $3 \cdot 2$ ), and was placed in a cot with a thin sheet and duvet (total tog count 9.0). The room temperature was stable, and averaged $19 \cdot 5^{\circ} \mathrm{C}$ for most of the night. During the three hours of recording he slept quietly without turning, and seemed restful and comfortable. There was no visible sweating. Figure 1 shows his rectal temperature during the night, and fig 2 shows drawings from thermographic images taken 30 minutes and two hours after bedtime, respectively. At bedtime, when his rectal temperature was above $37^{\circ} \mathrm{C}$, part of his head was warm (about $33^{\circ} \mathrm{C}$ ),

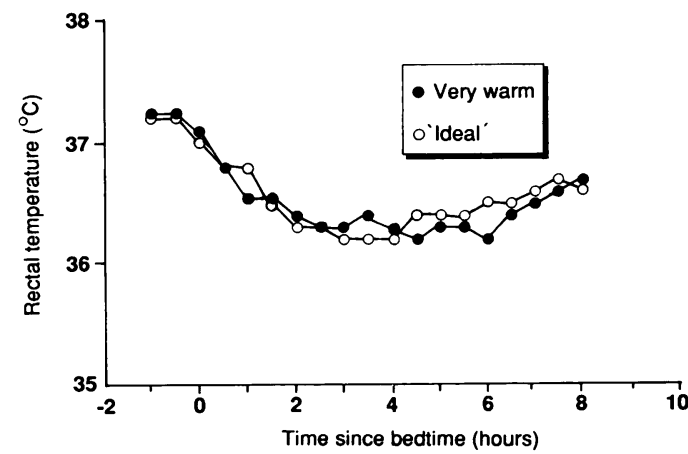

Figure 1 Rectal temperatures of case 1 (ideal) and case 2 (too warm) during the night. 


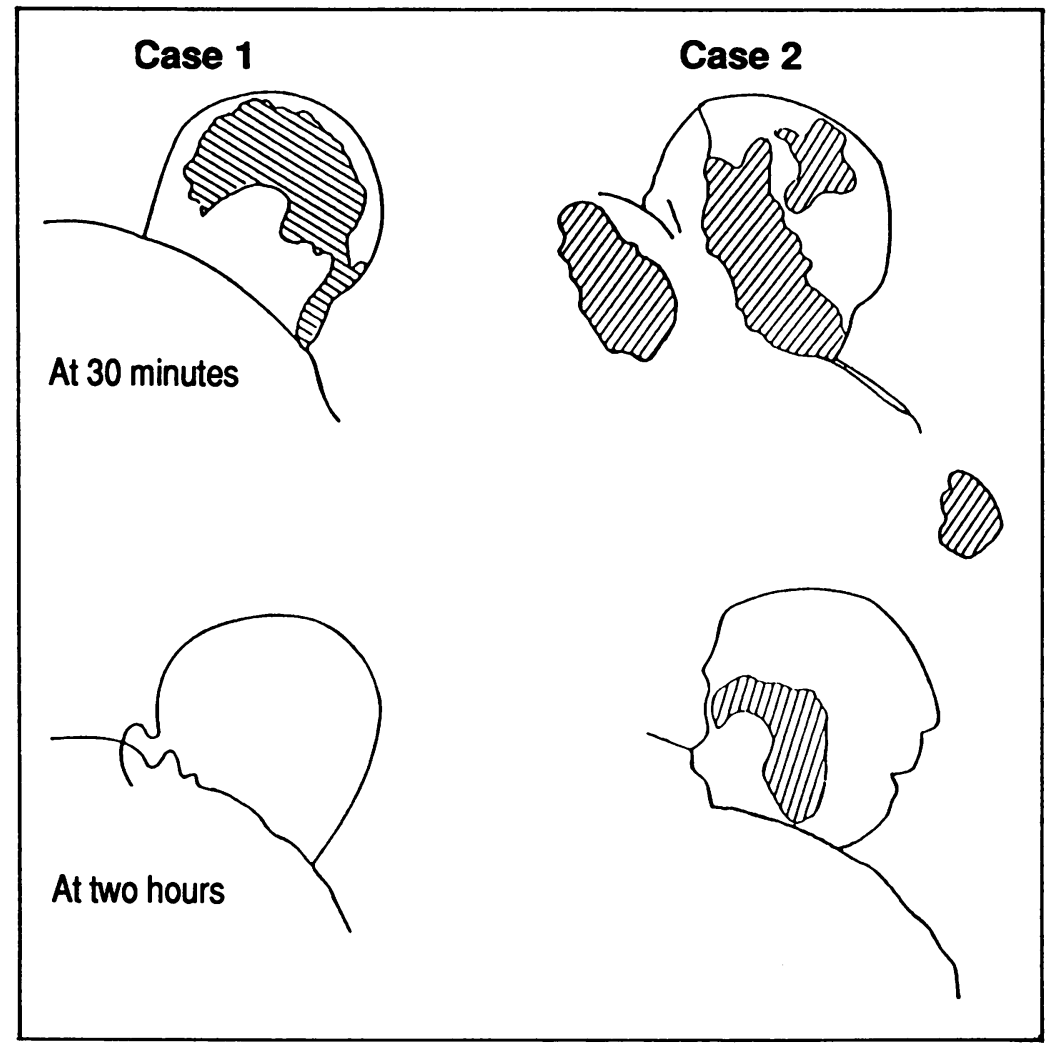

Figure 2 Thermographic images at 30 minutes and two hours after the babies had been put to bed, showing areas of maximum heat loss (shaded). Case 2 is using her hands as supplementary 'radiators'. graphic images at 30 minutes and two hours after bedtime, respectively. Her head and hands remained warm throughout with the surface thermistor registering $33^{\circ} \mathrm{C}$, and as she moved around she was continually exposing different parts of her head. After three hours, when her rectal temperature had cooled to $36 \cdot 3^{\circ} \mathrm{C}$ she withdrew her hands, and her head cooled a little, though it remained much hotter than that of case 1. Both babies had the same head temperature at bedtime. Neither baby woke or cried during the time of recording, though case 2 did wake later in the night.

\section{Discussion}

These cases are a testament to the capabilities of infants for thermoregulation, and the possibility of using thermographic imaging at home to show heat loss and sleeping body movements in different home conditions. Case 1 was kept in what many would believe to be a near perfect thermal environment, lightly wrapped at $19^{\circ} \mathrm{C}$. Case 2 would be described as 'over wrapped' by most observers, and slept in a decidedly warm room. Their night time patterns of rectal temperature were, however, identical and corresponded very closely to the normal pattern we have described previously. ${ }^{1}$

Whatever assumptions are made, it is difficult to see how case 2 could be losing any appreciable amount of heat from her heavily insulated body, and indeed her abdominal skin temperature and rectal temperature was almost identical, suggesting minimal heat flux. By some means, therefore, she lost sufficient heat through her head and hands to cool normally during sleep. This was achieved in part by increasing the exposed area as she moved around, which may indicate a considerable role for behaviour in infant thermoregulation. She was also sweating profusely, and the head and particularly hand surface temperatures were high, suggesting a considerable heat flux that was presumably attributable to increased cutaneous perfusion.

Although we have reported only two cases, we believe that they clearly show the need to study in much more detail the role of head, hands, and other parts of the body in the thermoregulation of infants under home conditions and the effects of different sleeping positions on temperature control and other physiological systems. Above all, they confirm the feasibility of detailed study of babies at home. pulled well up to her head. The room tem ture was $25 \cdot 2{ }^{\circ} \mathrm{C}$ at bedtime, and averaged $23.6^{\circ} \mathrm{C}$ during the three hours of thermal imaging, cooling only slightly for the rest of the night.

During the three hours of recording she was restless, and within 20 minutes had moved to expose more of her head, and removed her hands from the covers. She was obviously sweating profusely on the forehead and face. Figure 1 shows her rectal temperature during the night, which was indistinguishable from that of case 1 . Figure 2 shows her thermo-
We thank the parents for allowing us to study their babies and intrude into their homes. We are grateful to Insight Visions for the loan of the camera, and the Foundation for the Study of Infant Deaths for funding the research health visitor.

1 Wailoo MP, Petersen SA, Whitaker H, Goodenough P. Sleeping body temperature of $3-4$ month old infants. Arch Dis Child 1989;64:596-9.

2 Wailoo MP, Petersen SA, Whitaker H, Goodenough P. The thermal environment in which $3-4$ month old infants sleep at home. Arch Dis Child 1989;64:600-4.

3 Clark RP, Stothers JK. Neonatal skin temperature distribution using infra-red colour thermography. $\mathcal{F}$ Physiol 1980 302:323-33. 